\title{
Maculopathy in patients with diabetes mellitus type 1 and type 2: associations with risk factors
}

\author{
Eckhard Zander, Sabine Herfurth, Beate Bohl, Peter Heinke, Uwe Herrmann,
} Klaus-Dieter Kohnert, Wolfgang Kerner

\begin{abstract}
Aim-To examine possible relation between diabetic maculopathy and various risk factors for diabetic complications in patients with diabetes mellitus type 1 and type 2.

Methods-Cross sectional study of two cohorts of diabetic patients, comprising 1796 patients with type 1 diabetes (mean age 47 years, mean duration of diabetes 24 years) and 1563 patients with type 2 diabetes (mean age 62 years, mean duration of diabetes 16 years). Retinopathy levels (R0-RV) and maculopathy were assessed by fluorescence angiography and fundus photography and binocular biomicroscopy. Diabetic neuropathy was assessed by means of computer assisted electrocardiography and by thermal and vibratory sensory examination. Patients were classified as normoalbuminuric ( $<20 \mu \mathrm{g} / \mathrm{min})$ or microalbuminuric (20-200 $\mu \mathrm{g} / \mathrm{min}$ ) according to their albumin excretion rates measured in urine collected overnight. Using univariate analyses, the effects of selected patient characteristics on the presence of maculopathy were evaluated. Multiple logistic regression analyses were performed to determine independent effects of risk variables on diabetic maculopathy.
\end{abstract}

Results-Background retinopathy (RII) was found to be present in $28 \%$ of type 1 diabetic patients and in $38 \%$ of type 2 diabetic patients. The prevalence of maculopathy in these patients was remarkably high $(42 \%$ in type 1 and $53 \%$ in type 2 diabetic patients). Patients with maculopathy had significantly impaired visual acuity. Multiple logistic correlation analysis revealed that in both types of diabetes maculopathy exhibited independent associations with duration of diabetes and with neuropathy ( $p 0.01$ ); in type 1 diabetic patients there were significant associations with age at diabetes onset, serum triglyceride and total cholesterol levels (p $<0.05$ ); in type 2 diabetes with serum creatinine levels and with hypertension ( $p$ $<0.05$ ).

Conclusions-Irrespective of the type of diabetes, diabetic patients with long standing diabetes have a high risk for the development of diabetic maculopathy. Diabetic maculopathy is closely associated with diabetic nephropathy and neuropathy and with several atherosclerotic risk factors which suggests that these factors might have an important role in the pathogenesis of maculopathy. However, prospective trials are necessary to evaluate the predictive value of such factors. The findings of the present cross sectional study reinforce the arguments of previous studies by others for tight control of hypertension and hyperglycaemia.

(Br f Ophthalmol 2000;84:871-876)

Diabetic retinopathy is the most common microvascular complication in diabetes which can produce severe visual loss. ${ }^{12}$ The pathogenetic mechanisms involved in the onset and progression of retinopathy are poorly understood. ${ }^{3}{ }^{4}$ Independent of diabetic retinopathy, severe visual impairment among diabetic patients may also be caused by diabetic maculopathy. Diabetic maculopathy, resulting from diabetic retinopathy, is defined as the presence of retinal thickening within one disc diameter or two of the macula..$^{5-8}$ Macular oedema results from the accumulation of fluid at the posterior pole of the retina and visual acuity is threatened if the retina in the centre of the macula is thickened. ${ }^{9}$ Factors associated with the development of maculopathy are mostly unknown. ${ }^{8}{ }^{10}$ Since diabetic maculopathy is characterised by increased capillary leakage in the main retinal vessels and by alterations in the microcirculation of the macula, ${ }^{11}{ }^{12}$ several previous reports have suggested that poor metabolic control might be involved in haemodynamic changes of retinal circulation, and thereby lead to maculopathy. It is conceivable that increases in the retinal blood flow could play a part in haemodynamic changes of increased intracapillary retinal pressure and shear stress, thereby leading to diabetic maculopathy. ${ }^{3}{ }^{13} 14$

Most studies about diabetic maculopathy and associated risk factors are hospital based cohort studies. ${ }^{15-18}$ There is only one population based ${ }^{10}$ cohort study which was extended to a longitudinal study. ${ }^{19}$ Recent data have shown that better glycaemic and blood pressure control are beneficial in reducing the incidence of macular oedema. ${ }^{19}$

It was the aim of present cross sectional study (1) to determine the prevalence of diabetic maculopathy in both type 1 and type 2 diabetic patients and (2) to study the associations between diabetic maculopathy and different risk factors.

\section{Research design and methods} PATIENTS

The present cross sectional study, based on the total clinic population, included 1796 nonselected type 1 diabetic patients and 1563 type
Accepted for publication 24 February 2000 
2 diabetic patients with and without insulin treatment, attending our hospital during 1995 and 1996. All patients were white people residing in the northeastern region of Germany. Diagnosis of type 1 or type 2 diabetes was made by clinical judgment (age at diagnosis; start of insulin treatment during the first year after diagnosis). When diagnosis was uncertain, the result of $\mathrm{C}$ peptide measurement was included into diagnostic decision. Clinical characteristics of the patients studied are shown in Table 1. All patients gave their informed consent and signed an agreement for diagnosis, assessments, photographs, and fluorescein angiography according to the declarations of Helsinki and Hong Kong.

\section{STUDY DESIGN}

Assessment of diabetic retinopathy

Ophthalmoscopic examinations in all patients were carried out by two experienced ophthalmologists. Grading of diabetic retinopathy was done after ophthalmoscopy with dilated pupils and drawing the ophthalmoscopic picture and by fluorescence angiographic investigation. Retinopathy was determined according to Seidlein and Herfurth ${ }^{20}$ and was classified as follows:

$\mathrm{R} 0$ = without retinopathy

Non-proliferative retinopathy

$\mathrm{RI}=$ microaneurysms only

RII = minor retinal haemorrhages, hard and/or soft exudates (cotton wool exudates, several microaneurysms) = background retinopathy

Proliferative retinopathy

RIII = new vessels and/or fibrous proliferations without involving corpus vitreous

RIV = papillary neovascularisation, proliferative retinopathy involving corpus vitreous with rubeosis iridis

$\mathrm{RV}=$ blindness due to diabetic retinopathy (visus $<0.15=6 / 90$ ).

\section{Assessment of diabetic maculopathy}

Clinically significant diabetic maculopathy was defined in accordance with the ETDR Study ${ }^{21}$ by the presence of a set of characteristics:

- retinal thickening at or within $500 \mu \mathrm{m}$ of the centre of the macula
- hard exudates at or within $500 \mu \mathrm{m}$ of the macula, if associated with thickening of adjacent retina, or

- a zone, or zones, of retinal thickening one disc diameter or larger, any part of which is within one disc diameter of the centre of the macula.

Examinations of diabetic maculopathy were carried out by ophthalmologists by stereo slit lamp biomicroscopy, fundus photography, and fluorescein angiography. It should be noted that diabetic macular oedema is only one of the alterations of the macula and this term is not synonymous with diabetic maculopathy. Diabetic maculopathy includes focal macular oedema, diffuse macular oedema, cystoid macular oedema, exudative macular oedema, ischaemic maculopathy, and macular oedema of proliferative diabetic retinopathy. ${ }^{22}$

\section{Assessment of diabetic neuropathy and} hypertension

Cardiovascular autonomic neuropathy was diagnosed by measurement of $R R$ intervals during rest, deep breathing, and Valsalva manoeuvre by means of computer assisted one lead ECG ${ }^{23}$ (ProSciCard; Pro Science, Private Research Institute $\mathrm{GmbH}$, Germany). Data were recorded by EIZO 4050 Monitor (EIZO Corporation, Japan). Blood pressure response was tested in a supine position and 1 minute after standing up. A decrease in systolic blood pressure of more than $30 \mathrm{~mm} \mathrm{Hg}$ was considered as abnormal. Diagnosis of autonomic neuropathy was made when two or more of these tests were abnormal.

Diagnosis of peripheral polyneuropathy was made by clinical judgment and was based on symptoms (pain, paraesthesia) and results of clinical examination (ankle tendon reflexes, perception of vibration measured with a 128 $\mathrm{Hz}$ tuning fork, temperature sensation).

Arterial hypertension was diagnosed in patients with a history of antihypertensive drug treatment or with blood pressure values higher than 140/90 mm Hg. Nine blood pressure measurements were performed throughout a day in the sitting position after 5 minutes of rest. In addition, in 670 patients 24 hour blood pressure measurements were performed. In these patients hypertension was diagnosed if

Table 1 Clinical characteristics of type 1 and type 2 diabetic patients

\begin{tabular}{|c|c|c|c|c|c|c|c|c|}
\hline & \multicolumn{4}{|c|}{ Type 1 diabetes $(n=1796)$} & \multicolumn{4}{|c|}{ Type 2 diabetes $(n=1563)$} \\
\hline & \multirow[b]{2}{*}{$R 0-I$} & \multicolumn{2}{|l|}{$R I I-I V$} & \multirow[b]{2}{*}{$R V$} & \multirow[b]{2}{*}{$R O-I$} & \multicolumn{2}{|l|}{$R I I-I V$} & \multirow[b]{2}{*}{$R V$} \\
\hline & & without $M P$ & with $M P$ & & & without $M P$ & with $M P$ & \\
\hline No of patients & 1101 & 406 & 269 & 20 & 869 & 320 & 362 & 12 \\
\hline Age (years) & $28.3(0.4)$ & $44.3(0.6)$ & $46.6(0.8)$ & $48.2(2.6)$ & $59.2(0.4)$ & $61.1(0.5)$ & $62.1(0.4)$ & $71.2(3.1)$ \\
\hline Diabetes duration (years) & $9.1(0.2)$ & $26.6(0.5)$ & $25.1(0.6)$ & $33.2(1.5)$ & $9.4(0.2)$ & $16.1(0.4)$ & $17.6(0.3)^{\star}$ & $19.3(2.9)$ \\
\hline Age at manifestation (years) & $19.8(0.4)$ & $18.2(0.6)$ & $21.7(0.8)^{\star \star}$ & $15.4(2.9)$ & $50.3(0.4)$ & $45.5(0.6)$ & $44.7(0.5)$ & $51.8(5.1)$ \\
\hline $\mathrm{BMI}\left(\mathrm{kg} / \mathrm{m}^{2}\right)$ & $23.4(0.1)$ & $25.3(0.2)$ & $25.8(0.3)$ & $25.3(0.8)$ & $30.0(0.2)$ & $30.2(0.3)$ & $30.2(0.3)$ & $29.1(1.0)$ \\
\hline $\operatorname{AER}(\mu \mathrm{g} / \mathrm{min})$ & $23.0(3.1)$ & $131.4(16.2)$ & $126.3(18.2)$ & $286.1(128.1)$ & $69.4(6.6)$ & $208.7(30.0)$ & $224.8(22.9)$ & $247.8(99.1)$ \\
\hline Creatinine $(\mu \mathrm{mol} / \mathrm{l})$ & $73.3(0.7)$ & $88.0(2.6)$ & $99.4(4.5)^{\star \star}$ & $139.5(29.8)$ & $82.8(0.9)$ & $100.3(4.3)$ & $101.4(2.9)$ & $108.4(13.4)$ \\
\hline $\mathrm{Tg}(\mathrm{mmol} / \mathrm{l})$ & $1.5(0.05)$ & ) $1.5(0.05)$ & $1.9(0.1)^{\star}$ & $1.4(0.1)$ & $3.3(0.1)$ & $3.3(0.2)$ & $3.1(0.1)$ & $2.2(0.2)$ \\
\hline Cholesterol $(\mathrm{mmol} / \mathrm{l})$ & $5.2(0.04)$ & $5.8(0.06)$ & $6.2(0.1)^{\star \star}$ & $5.7(0.28)$ & $6.4(0.06)$ & $6.5(0.11)$ & $6.6(0.10)$ & $6.4(0.23)$ \\
\hline $\mathrm{HbA}_{1 \mathrm{c}}(\%)$ & $8.7(0.06)$ & $8.5(0.08)$ & $8.6(0.1)$ & $8.4(0.31)$ & $9.2(0.06)$ & $9.1(0.10)$ & $9.3(0.09)$ & $9.3(0.51)$ \\
\hline
\end{tabular}

Data represent means (SEM) and differences between RII-IV with maculopathy and RII-RIV without maculopathy (MP) significant at $\mathrm{p}<0.05$ and $\mathrm{p}<0.01$, respectively.

Significance ${ }^{\star \star} \mathrm{p}<0.01 ;{ }^{\star} \mathrm{p}<0.05$.

$\mathrm{n}=$ total number of patients in each cohort. 
mean blood pressure value was higher than 135/85 mm Hg.

Assessment of diabetic nephropathy

Diabetic nephropathy was diagnosed when albumin excretion rates exceeded $200 \mu \mathrm{g} / \mathrm{min}$ and/or serum creatinine levels exceeded 102 $\mu \mathrm{mol} / 1$ in male and $88 \mu \mathrm{mol} / 1$ in female patients, respectively. Normoalbuminuria was defined as albumin excretion rate $<20 \mu \mathrm{g} / \mathrm{min}$, microalbuminuria as albumin excretion rate between 20.0 and $200 \mu \mathrm{g} / \mathrm{min}$.

BIOCHEMICAL METHODS

Urinary albumin excretion rates (UAE) were measured by immunoturbidimetric method (Cobas Mira, Hoffmann La Roche AG) ${ }^{24}$ in urine samples collected on three occasions from $10 \mathrm{pm}$ to $6 \mathrm{am}$. Serum for measurement of cholesterol (CHOD-PAP method), triglycerides (GPO-PAP method), HDL-cholesterol (phosphotungstate magnesium method), and creatinine (Jaffé method) was prepared from blood samples drawn after an overnight fast; measurements were performed with a Hitachi911 analyser (Hoffmann La Roche AG) or Cobas Mira Analyser (Hoffmann La Roche AG). Serum C peptide was measured by ELISA (Dako-Diagnostica $\mathrm{GmbH}$ ) in fasting serum samples. $\mathrm{HbA}_{1 \mathrm{c}}$ was measured in haemolysed EDTA-blood by HPLC method (Diamat, Bio-Rad Laboratories).

\section{STATISTICAL METHODS}

Data are presented as means (SEM). Statistical significance was tested by $t$ test or $\chi^{2}$ test as indicated in the corresponding tables. Stepwise multiple logistic regression analysis (SPSS, Version 9.0) was applied to evaluate the impact on manifestation of diabetic maculopathy.

Diabetes duration, age at disease onset, $\mathrm{BMI}$, insulin requirement/body weight, $\mathrm{HbA}_{1 \mathrm{c}}$, serum creatinine, triglyceride, cholesterol, HDL cholesterol, hypertension, neuropathy, and nephropathy were considered as risk variables for diabetic maculopathy. They were coded as indicator variables-that is, representing the effect of each category compared with reference category of low levels.

\section{Results}

The prevalence of diabetic maculopathy was determined to be $15 \%$ in patients with type 1 and $23 \%$ in patients with type 2 diabetes. In type 1 diabetic patients $28 \%(500 / 1769)$ had background retinopathy. In those patients with background retinopathy 42\% (208/500) had diabetic maculopathy. Among type 1 diabetic patients $10 \%(175 / 1796)$ were suffering from proliferative retinopathy and, of those, 35\% (61/175) had diabetic maculopathy. In type 2 diabetic patients 38\% (598/1563) had background retinopathy and 53\% (315/598) diabetic maculopathy. In the group of type 2 diabetic patients $(5 \% ; 84 / 1563)$ with proliferative retinopathy $56 \%(47 / 315)$ had diabetic maculopathy. Type 1 diabetic patients with maculopathy were characterised by higher mean serum creatinine (99.4 (4.5) versus 88.0 (2.6) $\mu \mathrm{mol} / \mathrm{l} ; \mathrm{p}<0.01)$, higher mean triglyceride (1.9 (0.1) versus $1.5(0.1) \mathrm{mmol} / \mathrm{l} ; \mathrm{p}<0.05)$, and higher mean serum cholesterol levels $(6.2(0.1)$ versus $5.8(0.1) \mathrm{mmol} / \mathrm{l} ; \mathrm{p}<0.01)$ than those patients without maculopathy (Table 1). In type 2 diabetic patients no such differences were found and, as in the in the type 1 diabetic cohort, there were neither differences in mean albumin excretion rates nor in $\mathrm{HbA}_{1 \mathrm{c}}$ levels between patients with and without diabetic maculopathy (Table 1). Visual acuity decreased with increasing severity of diabetic retinopathy $(\mathrm{p}<0.01)$ in type 1 and type 2 diabetic patients (Table 2). In either type of diabetes with maculopathy, the distribution between retinopathy levels was similar for both eyes. Prevalence rates of maculopathy in cohorts with type 1 and type 2 diabetes were found to increase consistently with diabetes duration up to 30 years (Table 3), but tended to be higher in type 2 than in type 1 diabetes. No relation was found between the prevalence of maculopathy and levels of glycosylated haemoglobin. Compared with patients without diabetic maculopathy, increased prevalences in either type of diabetes were associated with hypertension, nephropathy, and neuropathy (Table 3). Multiple regression analysis, performed to evaluate whether maculopathy was independently associated with characteristic variables or other diabetic complications (Table 4), revealed significant associations with the presence of peripheral and/or autonomic neuropathy $(p<0.01)$ in both type 1 and type 2 diabetic patients. Another independent association included diabetes duration $(p<0.01)$. Differences in associations of maculopathy and patient characteristics were found between type 1 and type 2 diabetic patients. In the type 1 diabetic cohort, maculopathy was positively associated with the age at disease onset $(\mathrm{p}<0.05)$, increased triglyceride levels $(\mathrm{p}<0.05)$, and increased cholesterol levels $(\mathrm{p}<0.05)$. Non-significant associations included increased creatinine levels and hypertension. In contrast, the type 2 diabetic cohort exhibited independent associations of macu-

Table 2 Impact of retinopathy and maculopathy (MP) on visual acuity in diabetic patients

\begin{tabular}{llllll}
\hline \multirow{2}{*}{ Level of diabetic retinopathy } & \multicolumn{2}{l}{ Type 1 diabetes $(n=1796)$} & & \multirow{2}{*}{ Type 2 diabetes $(n=1563)$} \\
\cline { 2 - 3 } & Right & Left & & Right & Left \\
\hline (a) 0/I & $0.90(0.19)^{\star \star 1}$ & $0.90(0.18)^{\star \star 1}$ & & $0.72(0.24)$ & $0.72(0.24)$ \\
(b) II-IV without MP & $0.72(0.29)^{\star \star 2}$ & $0.73(0.27)^{\star \star 2}$ & & $0.62(0.27)^{\star \star 1}$ & $0.64(0.27)^{\star \star 1}$ \\
(c) II-IV with MP & $0.62(0.28)^{\star \star 3}$ & $0.59(0.30)^{\star \star 3}$ & & $0.51(0.27)^{\star \star 2}$ & $0.51(0.28)^{\star \star 2}$ \\
(d) V & $0.30(0.40)$ & $0.22(0.33)$ & & $0.01(0.03)^{\star \star 3}$ & $0.14(0.33)^{\star \star 3}$ \\
\hline
\end{tabular}

Data are means $(\mathrm{SD}) ; \mathrm{n}=$ number of patients.

Significance: ${ }^{\star \star} \mathrm{p}<0.01$

Type 1 diabetes: (1) $v$ b, c, d, (2) $v$ c, d, (3) $v$ d. Type 2 diabetes: (1) $v$ a, (2) $v$ a, b, (3) $v$ a, b, c. 
Table 3 Prevalence of different risk variables in type 1 and type 2 diabetics with maculopathy

\begin{tabular}{lcc}
\hline Variable & Type 1 diabetes & Type 2 diabetes \\
\hline Age at manifestation (years) & \\
$\quad 0-19$ & 12.1 & \\
$20-39$ & 19.7 & 29.4 \\
$40-59$ & 18.7 & 25.0 \\
$>60$ & 11.5 & 9.3 \\
Diabetes duration (years) & & \\
$0-9$ & 1.5 & 6.0 \\
10-19 & 16.4 & 27.6 \\
$20-29$ & 30.7 & 45.2 \\
$>30$ & 28.1 & 37.2 \\
HbA & & \\
$>6.5$ & & 19.1 \\
$6.5-8.0$ & 15.3 & 23.3 \\
$>8.0$ & 14.7 & 23.5 \\
Hypertension & 25.1 & $26.1(3.3)$ \\
Nephropathy & $35.6(14.3)$ & $43.2(21.9)$ \\
Neuropathy & $31.4(6.2)$ & $30.3(10.5)$ \\
Tg $>2.2$ mmol/1 & $21.9(13.8)$ & $23.1(23.1)$ \\
Chol >6.5 mmol/1 & $26.0(12.4)$ & $24.4(22.2)$ \\
\hline
\end{tabular}

The prevalence rates (\%) for patients without maculopathy are given in parentheses for comparison.

lopathy with elevated serum creatinine levels $(p<0.05)$ and hypertension $(p<0.05)$, while non-significant associations included age at disease onset, serum triglyceride, and cholesterol levels.

\section{Discussion}

The present cross sectional study shows that maculopathy occurs frequently in type 1 and type 2 diabetic patients. Prevalence was higher in type 2 than in type 1 diabetic patients. The majority of these patients suffered from background retinopathy. In type 2 diabetes with proliferative retinopathy, the proportion of patients with maculopathy is found to be higher than in type 1 diabetes with proliferative retinopathy. Since the data of the present cross sectional study were obtained from a selected patient population, they cannot directly be compared with data from population based epidemiological studies. Nevertheless, it is noteworthy that the prevalence of proliferative retinopathy in our study was found to be $10 \%$ in type 1 diabetic patients and 5\% in type 2 diabetic patients. These data match with findings of the Wisconsin Epidemiologic Study of Diabetic Retinopathy, where the overall incidence of retinopathy was reported to be $10 \%$ and $7 \%$ among the younger and older onset group. ${ }^{2}$ The prevalence of background retinopathy in our study is rather high, $28 \%$ in

Table 4 Multiple logistic correlation analysis (odds ratios) for maculopathy in type 1 and type 2 diabetes

\begin{tabular}{lll}
\hline & Patients \\
\cline { 2 - 3 } Variable & $\begin{array}{c}\text { Type 1 diabetes } \\
(n=1796)\end{array}$ & $\begin{array}{c}\text { Type 2 diabetes } \\
(n=1563)\end{array}$ \\
\hline Age at manifestation (years) & & \\
$\quad 40-60$ & $2.1^{\star}$ & \\
$>60$ & $5.0^{\star}$ & \\
Diabetes duration (years) & $9.1^{\star \star}$ & $3.7^{\star \star}$ \\
$\quad 10-20$ & $15.3^{\star \star}$ & $7.2^{\star \star}$ \\
$20-30$ & $10.1^{\star \star}$ & $5.3^{\star \star}$ \\
$>30$ & $1.5^{\star}$ & \\
Tg $>2.2$ mmol/1 & $1.6^{\star}$ & $1.3^{\star}$ \\
Chol $>6.5$ mmol/1 & & $1.5^{\star}$ \\
Elevated creatinine & $3.5^{\star \star}$ & $3.1^{\star \star}$ \\
Hypertension & Peripheral and/or autonomic neuropathy &
\end{tabular}

Significance: ${ }^{\star \star} \mathrm{p}<0.01,{ }^{\star} \mathrm{p}<0.05, \mathrm{n}=$ number of patients. type 1 and $38 \%$ in type 2 diabetic patients. Furthermore, the prevalence of maculopathy in our type 1 diabetic cohort is $15 \%$ and in the type 2 diabetic cohort $23 \%$, whereas the prevalence of macular oedema of the Wisconsin Epidemiologic Study of Diabetic Retinopathy was reported to be $11 \%$ in the younger onset group and $8 \%$ in the older onset group. ${ }^{21}$ The prevalence rates we found are higher than those from population based studies because of patient selection. ${ }^{21}$ However, other cross sectional and clinical studies ${ }^{16}{ }^{18} 25$ have reported similar frequencies for diabetic maculopathy to our study. In most previous studies, higher rates of macular oedema were found in the presence of non-proliferative retinopathy in older compared with younger onset patients. ${ }^{101726}$ The majority of our patients with retinopathy had background retinopathy. Among these patients the proportion of diabetic maculopathy is $42 \%$ in the type 1 diabetic and $53 \%$ in the type 2 diabetic cohort. The proportion of maculopathy is similarly high in those patients with proliferative retinopathy, being $35 \%$ and $56 \%$ for type 1 and type 2 diabetic patients. In either type of diabetes the presence of maculopathy is associated with diabetes duration and in type 1 diabetic subjects with older age at disease manifestation. These findings are consistent with the Wisconsin Epidemiologic Study of Retinopathy data, demonstrating an increased prevalence of diabetic maculopathy of $28 \%$ in patients whose age at the time of diagnosis was 30 years or older and whose diabetes duration was 20 years or longer. In these cases the presence of macular oedema was associated with longer duration of diabetes, higher systolic blood pressure, higher levels of glycosylated haemoglobin and with the presence of proteinuria. $^{10}$

Our data collected from large cohorts of hospital patients confirm the impact of diabetic maculopathy on visual acuity. Regardless of the type of diabetes, the visual acuity of patients with maculopathy is significantly impaired compared with patients without maculopathy, although at identical stages of diabetic retinopathy. ${ }^{9} 27$

After 14 years of observation the Wisconsin Epidemiologic Study of Diabetic Retinopathy showed an incidence of macular oedema of $26 \%$ in a cohort of insulin taking diabetic patients diagnosed before the age of 30 years. Furthermore, increased risk of proliferative retinopathy or incidence of macular oedema was found to be associated with more baseline retinopathy, higher baseline $\mathrm{HbA}_{1}$, and increases in $\mathrm{HbA}_{1}$ levels during the follow up period. When hypertension was present at baseline it was associated with a $91 \%$ increase in the risk of proliferative retinopathy, while the presence of gross proteinuria at baseline was associated with a $95 \%$ increase in the risk of developing macular oedema. ${ }^{19}$ These data together with our present observation demonstrate that factors such as high $\mathrm{HbA}_{1}$ levels, high blood pressure, and proteinuria might contribute to macular oedema formation. 
Owing to the cross sectional nature of our data, relations that have been established from prospective studies - for example, between the incidence of macular oedema and glycaemic control, ${ }^{19}$ could not be identified in the our study. However, we found diabetic maculopathy in either type of diabetes to be independently associated with disease duration and thus with exposure to hyperglycaemia. Our study also shows significant associations of maculopathy with increased lipid levels in type 1 diabetic patients and with diabetic nephropathy and hypertension in type 2 diabetic patients. Even though we have not studied haemodynamic changes of the retina circulation in our patients, the present findings are consistent with data in the literature, providing evidence that increased arterial perfusion along with tissue hypoxia could produce macular oedema by exacerbating breakdown of the blood-retinal barrier. ${ }^{17} 2829$ In line with the association between diabetic maculopathy and nephropathy in type 2 diabetes is the higher frequency of macular oedema found by others in nephropathic diabetic patients with proteinuria. ${ }^{15}$ It is of particular interest that our study shows significant associations between maculopathy and neuropathy, taking into account peripheral as well as cardiovascular autonomic neuropathy. As with other diabetic complications, neuropathy in type 1 diabetes $^{30}{ }^{31}$ and maculopathy ${ }^{9}{ }^{10}$ shows associations with disease duration. On the other hand in a previous cross sectional study we found cardiovascular autonomic neuropathy to be closely related to diabetic retinopathy. ${ }^{32}$ This suggests that there may be a causal pathogenetic relation between these two microvascular complications. Although prospective epidemiological studies are needed to clarify whether or not neuropathy is predictive of diabetic retinopathy, ${ }^{33}$ there are clinical and experimental data, suggesting direct effects of autonomic nervous system on retinal blood flow ${ }^{12} 3^{35}$ and the occurrence of blood flow changes in diabetic retinopathy. ${ }^{36}$ These observations support the hypothesis of haemodynamic alterations as important factors in the pathogenesis of diabetic microangiopathy ${ }^{37}$ and diabetic maculopathy. Diabetic neuropathy could be implicated in the development of these complications, but it needs prospective clinical trials to confirm such a hypothesis. However, the underlying causes of diabetic maculopathy remain to a certain extent speculative and, definitively, nothing can be said without perfusion pressure measurements of retinal circulation. In summary, our present data are consistent with observations from a number of other studies ${ }^{19} 253839$ underlining the need for better glycaemic and blood pressure control in order to reduce the incidence of macular oedema and/or the progression of diabetic retinopathy. In addition, our data support the necessity of treating all type 1 and type 2 diabetic patients aggressively to achieve near normal glycaemic, lipid, and blood pressure values.
1 Klein R, Klein BEK, Moss SE, et al. The Wisconsin Epidemiologic Study of Diabetic Retinopathy III. Prevalence and isk of diabetic retinopathy when age at diagnosis is 30 or more years. Arch Ophthalmol 1984;102:527-32.

2 Klein R, Klein BEK, Moss SE. Epidemiology of proliferative diabetic retinopathy. Diabetes Care 1992;15:1875-91.

3 Grunwald JE, Du Pont J, Riva CE. Retinal haemodynamics in patients with early diabetes mellitus. $\mathrm{Br} \mathcal{F}$ Ophthalmol 1996;80:327-31.

4 Klein R, Moss SE, Klein BEK. New management concepts for timely diagnosis of diabetic retinopathy treatable by photocoagulation. Diabetes Care 1987;10:633-8.

5 Cunha-Vaz JG, Fairia de Abreu JR, Campos AJ, et al. Early breakdown of the blood-retina barrier in diabetes. $\mathrm{Br} F$ Ophthalmol 1975;59:649-56.

6 Cunha-Vaz JG, Travassos A. Breakdown of the blood-retina barriers and cystoid macular edema. Surv Ophthalmol 1984;28:485-92.

7 Ferris FL III, Davis MD, Aiello LM. Treatment of diabetic retinopathy. N Engl f Med 1999;341:667-78.

8 Ferris FL III, Patz A. Macular edema. A complication of diabetic retinopathy. Surv Ophthalmol 1984;28:452-61

9 Moss SE, Klein R, Klein BEK. The incidence of vision loss in a diabetic population. Ophthalmology 1988;95:1340-8.

10 Klein R, Klein BEK, Moss SE, et al. The Wisconsin Epidemiologic Study of Diabetic Retinopathy IV. Diabetic macular edema. Ophthalmology 1984;91:1464-74.

11 Arend O, Wolf S, Jung F, et al. Retinal microcirculation in patients with diabetes mellitus: dynamic and morphologi$\mathrm{cal}$ analysis of perifoveal capillary network. Brf Ophthalmol 1991;75:514-18.

12 Grunwald JE, Riva CE, Baine J, et al. Total retinal volumetric blood flow rate in diabetic patients with poor metabolic control. Invest Ophthalmol Vis Sci 1992;33:356-33.

13 Kohner EM, Hamilton AMP, Saunders SJ, et al. The retinal Kohner EM, Hamilton AMP, Saunders SJ, et al. The

14 Patel V, Rassam S, Newsom R, et al. Retinal blood flow in diabetic retinopathy. BMF 1992;305:678-83.

15 Aiello LM, Rand LI, Briones JC, et al. Nonocular clinical risk factors in the progression of diabetic retinopathy. In: Little HL, Jack RL, Patz A, Forsham PH, eds. Diabetic retinopathy. New York: Thieme-Stratton, 1983:21-31.

16 British Multicenter Study Group. Photocoagulation for diabetic maculopathy: a randomized controlled clinical trial using the xenon arc. Diabetes 1983;32:1010-16.

17 Klein BEK, Moss SE, Klein R, et al. The Wisconsin Epidemiologic Study of Diabetic Retinopathy XIII. Relationship of serum cholesterol to retinopathy and hard exudates. Ophthalmology 1991;98:1261-5.

18 Myers FL, Davis MD, Magli YL. The natural course of diabetic retinopathy: a clinical study of 321 eyes followed one year or more. In: Goldberg MF, Fine SL, eds. Symposium on the treatment of diabetic retinopathy. Airlie House, Warrenton, Virginia, 29 September to 1 October 1968. Warrenton, Virginia, 29 September to 1 October 1968. Washington DC: US Govern

19 Klein R, Klein BEK, Moss SE, et al. The Wisconsin Epidemiologic Study of Diabetic Retinopathy XVII. The 14-year incidence and progression of diabetic retinopathy and associated risk factors in type 1 diabetes. Ophthalmology 1998;105:1801-5.

20 Seidlein I, Herfurth S. Klinisches Bild, Diagnostik, Klassifikation und Epidemiologie der diabetischen Retinopathie. Augenoptik (Berlin) 1990;107:7-9.

21 Early Treatment Diabetic Retinopathy Study Research Group. Photocoagulation for diabetic macular edema. Early Treatment Diabetic Retinopathy Study Report Number 1. Arch Ophthalmol 1985;103:1769-806.

22 Helbig H, Bornfeld N. Diagnostik und Therapie der diabetischen Retinopathie. Diabetes und Stoffwechsel 1997; 6:157-67.

23 Ziegler D, Laux G, Dannehl K, et al. Assessment of cardiovascular autonomic function: age-related normal ranges and reproducibility of spectral analysis, vector analysis and standard tests of heart rate variation and blood pressure responses. Diabet Med 1992;9:166-75.

24 Gottschling HD, Jahr D, Diaz-Alonso JM, et al. Enzymimmunoassay zur Bestimmung von Albumin im Urin. $Z$ med Labdiagn 1991;32:84-90.

25 Aiello LM, Rand LI, Briones JC, et al. Diabetic retinopathy in Joslin clinic patients with adult-onset diabetes. Ophthat mology 1981;88:619-23.

26 Klein R, Klein BEK, Moss SE, et al. The Wisconsin Epidemiologic Study of Diabetic Retinopathy XIV. Ten-year incidence and progression of diabetic retinopathy. Arch Ophthalmol 1994;112:1217-28.

27 Patz A, Schatz H, Berkow JW, et al. Macular edema-an overlooked complication of diabetic retinopathy. Trans Am Acad Ophthalmol Otolaryngol 1973;77:OP34-42.

28 Bresnick GH. Diabetic maculopathy: a critical review highlighting diffuse macular edema. Ophthalmology 1983;90: 1301-17.

29 Klein B, Klein R, Moss SE, et al. A cohort study of the relationship of diabetic retinopathy to blood pressure. Arch Ophthalmol 1995;113:601-6.

30 Maser RE, Steenkiste AR, Dorman JS, et al. Epidemiological correlates of diabetic neuropathy. Diabetes 1989;38: 1456-61.

31 Ziegler D, Grie $\beta$ FA, Spueler M, et al. The epidemiology of diabetic neuropathy. $\mathcal{F}$ Diabet Complications 1992;6:49-57.

32 Zander E, Heinke P, Herfurth S, et al. Relations between diabetic retinopathy and cardiovascular neuropathy. A cross-sectional study in IDDM and NIDDM patients. Exp Clin Endocrinol Diabetes 1997;105:19-26. 
33 Fong DS, Warram JH, Aiello LM, et al. Cardiovascular Fong DS, Warram JH, Aiello LM, et al. Cardiovascular autonomic neuropathy and proliferative
opathy. Am $\mathcal{f}$ Ophthalmol 1995;120:317-21.

34 Furukawa $H$. Autonomic innervation of preretinal blood Furukawa H. Autonomic innervation of preretinal blood vessels

35 Stephanson E, Landers MB, Wolbarsht ML. Oxygenation and vasodilatation in relation to diabetic and other proliferative retinopathies. Ophthalmic Surg 1983;14:209-26.

36 Feke GT, Buzney SM, Ogasawara H, et al. Retinal circulatory abnormalities in type 1 diabetes. Invest Ophthalmol Vis Sci 1994;35:2968.
37 Zatz R, Brenner BM. Pathogenesis of diabetic microangiopathy: the hemodynamic view. Am $\mathcal{F}$ Med 1986; 80:443

38 The DCCT Research Group. The effect of intensive treatment of diabetes on the development and progression of long-term complications in insulin-dependent diabetes mellitus. N Engl F Med 1993;329:977-86.

39 UK Prospective Diabetes Study (UKPDS) Group. Intensive blood-glucose control with sulfonylureas or insulin compared with conventional treatment and risk of complications in patients with type 2 diabetes (UKPDS 33). Lancet 1998;352:837-53. 